\title{
Interactive Manipulation Model of Group of Individual Bodies for VR Cooking System
}

\author{
Atsushi Morii, Daisuke Yamamoto, and Kenji Funahashi \\ Nagoya Institute of Technology, \\ Cokiso-cho, Showa-ku, Nagoya, 466-8555, Japan \\ morii@center.nitech.ac.jp, \\ yamamoto.daisuke@nitech.ac.jp, \\ kenji@nitech.ac.jp \\ http://www.center.nitech.ac.jp/
}

\begin{abstract}
A new high-speed manipulation model for a group of individual bodies (GIB) such as sand and lava is proposed in this paper. One of the goals of this research is to use it for applications such as home VR cooking systems for representing eg. a mass of fried rice. In this model, GIB is represented as a height field. Variation in the height field represents movement of the GIB. Transformation of GIB in wide spaces which is beyond adjacent grids is considered. GIB is treated as one object, which means that calculation is done efficiently on one object. Transformation of GIB is calculated quickly. Interactivity has a priority over correct movement of GIB in this model.
\end{abstract}

Keywords: a group of individual bodies, VR cooking system, home use.

\section{Introduction}

In recent years, research about interactive manipulation for sand and lava has been performed [1] 2]. In these researches, transformation of a group of individual bodies (GIB) such as lava and sand in a wide spaces which is beyond adjacent grids needs multiple calculations over a timeframe. For example, in a sand manipulation model [2], the collapse of the GIB is calculated by searching adjacent grids on every timeframe. The transformation of the GIB surface is conducted by sequences of calculations. In this paper, we propose a high-speed GIB model which only needs a few calculations for a certain point of time. In this model, we consider transformation of GIB in wide spaces which is beyond adjacent grids. GIB is treated as one object, which is calculated quickly.

\section{Manipulation Model of GIB}

Transformation Surface. In this model, GIB is defined as a height field which is fixed on a container. Transformation of the GIB is calculated from a curved surface. When the GIB exists in the center of a container, for example (Fig 1. 1 ), transformation surface (TS) is generated around place where the GIB seem 
to slide down to (Fig,1 2 the black part), and the volume is added to the GIB (Fig[1 3). Then the GIB volume is corrected (Fig[1 4), and as a result the GIB is moved. A TS is generated as a half of elliptic cylinder for simplified calculation [3]. For example, when the container is tilted, a TS is generated in Fig 2. When an acceleration is applied to the container, the GIB receives a reaction force. The TS is calculated from the gravity and the reaction force.

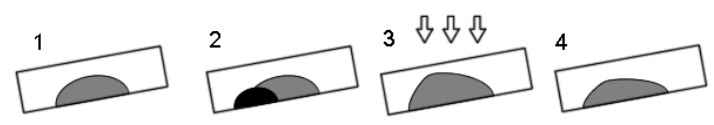

Fig. 1. Vertical Section of GIB and container

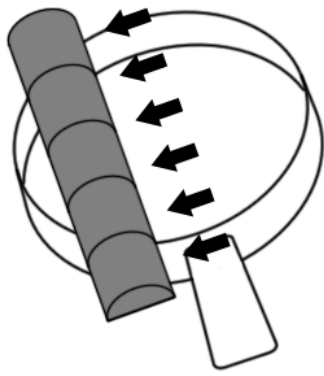

Fig. 2. TS of a half of elliptic cylinder

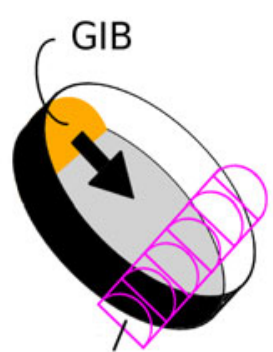

TS

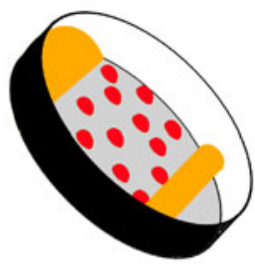

Fig. 3. Process of GIB movement

Expression Based on Existence Probability. Though TS model is quick because of its simple method, this model is insufficient in expressing the GIB's movement. To represent the process of GIB movement, we conduct an expression based on existence probability. When TS is generated on the place where TS is isolated from GIB in Fig 3 (left), we suppose that GIB are sliding down on the container. In this case, the GIB spreads both at the top of container and TS. Each grid of height field in the container are added random values based on existence probability (Fig 3 right). This expression is continued as long as more than a threshold volume exists between the center and the top of the container.

Particle System. We also implement a particle system [4] in this model. In order to implement the system, we locate the particles in height field values. Particle collision is detected on the surface of the height field. They also fall down when the container is tilted, and then the total volume of the GIB in the container is decreased. Only a few particles are used for maintaining a level of realism.

\section{Experiment and Conclusion}

The experiment was constructed on a system with a WiiRemote using a Intel Core 2 Duo E6300 1.86GHz CPU, 1024MB RAM. In this system, the GIB is 

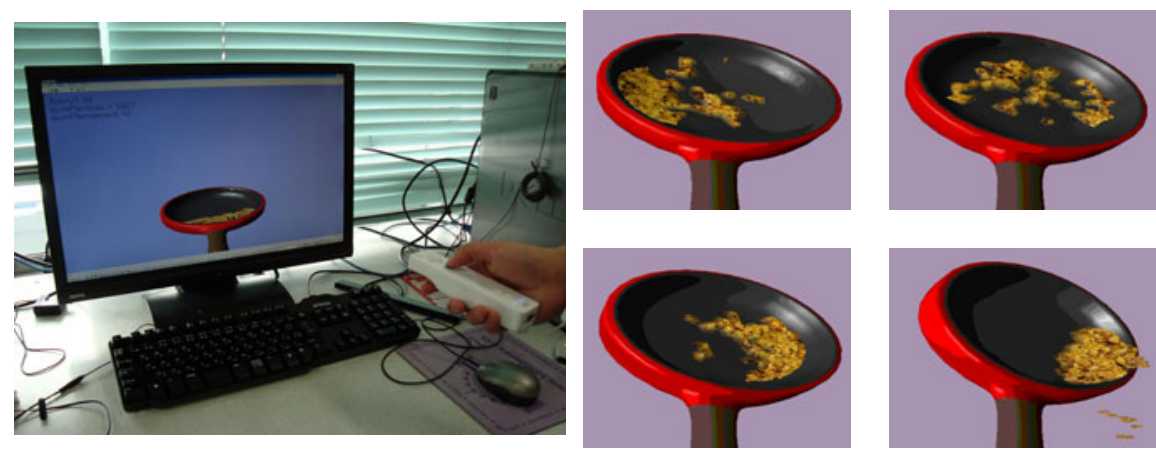

Fig. 4. System Appearance

supposed as fried rice (not traditional Chinese fried rice but Japanese) and the container as a skillet (Fig (4). Through this system, subjects were able to move fried rice by moving the container. 8 subjects evaluated the GIB movement's realism by classifying the score into seven grades. 1st grade is "This model doesn't feel real enough for a cooking system". 7th grade is "It is as natural as a real one". 3 subjects evaluated it as 4 . 4 subjects evaluated it as 5 . One subject evaluated it as 6 . The results of the performance test is shown in the Table 1 We got some positive evaluations such as that GIB movement seems natural with 331 vertices and 670 particles. Good results were obtained but there are some points that need improvement. We have to improve upward movement of the GIB without the particle system. We are also developing a model for using cooking tools e.g. a spatula in this model.

Table 1. Performance Results

\begin{tabular}{|c|c||c|}
\hline Vertices & Particles & FPS \\
\hline \hline 331 & 670 & 20 \\
\hline 631 & 1300 & 18 \\
\hline 817 & 1700 & 15 \\
\hline
\end{tabular}

\section{References}

1. Stora, D., Agliati, P.O., Cani, M.P., Neyret, F., Gascuel, J.D.: Animating lava flows. In: Proc. of the Graphics Interface, pp. 203-210 (1999)

2. Onoue, K., Nishita, T.: Virtual Sandbox. In: Proc. of the 11th Pacific Graphics, pp. 252-259 (2003)

3. Morii, A., Yamamoto, D., Funahashi, K.: Grid and particle based model of group of individual bodies for VR cooking system. In: Proc. of the 14th Virtual Reality Society of Japan Annual Conference (DVD) (2009) (in Japanese)

4. Harada, T., Tanaka, M., Koshizuka, S., Kawaguchi, Y.: Real-time Coupling of Fluids and Rigid Bodies. In: Proc. of the APCOM 2007-EPMESC XI (2007) 